TITLE:

\title{
A novel diagnostic criterion for lymph node metastasis in cervical cancer using multi-detector computed tomography.
}

\section{AUTHOR(S):}

Yamanoi, Koji; Matsumura, Noriomi; Kido, Aki; Baba, Tsukasa; Hamanishi, Junzo; Yamaguchi, Ken; Yoshioka, Yumiko; Taleb, Hisham Abou; Togashi, Kaori; Konishi, Ikuo

\section{CITATION:}

Yamanoi, Koji ...[et al]. A novel diagnostic criterion for lymph node metastasis in cervical cancer using multi-detector computed tomography.. Gynecologic oncology 2013, 131(3): 701-707

\section{ISSUE DATE:}

2013-12

URL:

http://hdl.handle.net/2433/179962

\section{RIGHT:}

(C) 2013 Elsevier Inc.; This is not the published version. Please cite only the published version.; この論文は出版社版でありません。引用の際に は出版社版をご確認ご利用ください。 
1 Original Article

2 A novel diagnostic criterion for lymph node metastasis in cervical cancer using

3 multi-detector computed tomography

4

5 Koji Yamanoi, $\mathrm{MD}^{\mathrm{a}}$, Noriomi Matsumura, $\mathrm{MD}, \mathrm{PhD}^{\mathrm{a}} \dagger$, Aki Kido, MD, $\mathrm{PhD}^{\mathrm{b}}$, Tsukasa Baba,

$6 \mathrm{MD}, \mathrm{PhD}^{\mathrm{a}}$, Junzo Hamanishi, MD, PhD ${ }^{\mathrm{a}}$, Ken Yamaguchi, MD, PhD ${ }^{\mathrm{a}}$, Yumiko Yoshioka, MD,

$7 \quad \mathrm{PhD}^{\mathrm{a}}$, Hisham Abou Taleb, $\mathrm{MD}^{\mathrm{a}}$, Kaori Togashi, $\mathrm{MD}, \mathrm{PhD}^{\mathrm{b}}$, Ikuo Konishi, $\mathrm{MD}, \mathrm{PhD}^{\mathrm{a}}$

8

$9 \quad{ }^{a}$ Department of Gynecology and Obstetrics, Kyoto University Graduate School of Medicine

$10{ }^{\mathrm{b}}$ Department of Diagnostic Imaging and Nuclear Medicine, Kyoto University Graduate

11 School of Medicine

12

$13 \uparrow$ Corresponding Author

1454 Shogoin Kawahara-cho, Sakyo-ku, Kyoto, 606-8507, Japan

15 Tel: $+81-75-751-3269$

16 Fax: +81-75-761-3967 (facsimile)

17 Email: noriomi@kuhp.kyoto-u.ac.jp 
19 Keywords: cervical cancer, lymph node metastasis, multi-detector computed tomography

20 (MDCT), neoadjuvant chemotherapy (NAC) 
ABSTRACT

Objectives: The sensitivity of the current $10 \mathrm{~mm}$ cut-off diameter that is used to diagnose

lymph node (LN) metastasis is too low. This is the first study to develop a new criterion to

diagnose LN metastasis in a region-by-region manner using multi-detector computed

tomography (MDCT).

Methods: 1) The short-axis diameter of the LNs in MDCT images from 1-mm slices

obtained immediately prior to surgery was compared with the pathological diagnosis in 78

uterine cervical cancer patients undergoing primary surgery. For the region-by-region

analysis, we divided para-aortic and pelvic spaces into 13 regions. 2) In 28 cases in which

patients received neoadjuvant chemotherapy (NAC) followed by surgery, we compared

MDCT images before and after NAC.

Results: 1) The optimal cut-off in the region-by-region analysis was $5 \mathrm{~mm}$, yielding $71 \%$

sensitivity and $79 \%$ specificity. 2) NAC significantly decreased $\mathrm{LN}$ size ( $\mathrm{p}<0.0001)$. NAC

decreased the number of swollen LN regions (>5 mm) from 51\% (81/158) to $26 \%(41 / 158)$.

Conclusions: The new criterion developed using MDCT could be effective for accurately assessing LN status. It also facilitates the assessment of NAC efficacy regarding the

eradication of LN metastases. 
Cervical cancer is the second most common gynecologic malignancy in the world [1].

In addition to surgery and radiotherapy, neoadjuvant chemotherapy (NAC) followed by

patients with cervical cancer depends largely on lymph node (LN) metastasis [3-5], it is

important to assess the LN status accurately before starting treatment.

Based on studies comparing the size of the LNs in images and pathological diagnoses,

a $10 \mathrm{~mm}$ short-axis diameter became the currently accepted cut-off in both clinical practice

and clinical trials [6-8]. However, the sensitivity of this conventional criterion has been

reported to be as low as $25 \%$ [9]. Furthermore, previous studies have generally predicted the

presence of LN metastases in the patient without describing the location. In the present study,

we conducted a detailed, region-by-region analysis to generate a new criterion that could

identify region-specific, swollen LNs. The use of our criterion could lead to the improvement

of individualized treatments.

In conducting the detailed analysis of LN status, the width of the slices, $7 \mathrm{~mm}$ in

used a multi-detector CT (MDCT) scanner, which allowed one mm slice images [10, 11]. 
57 Using MDCT, we successfully generated a new criterion to diagnose LN metastasis. Using

58 the new criterion, we were able to examine the efficacy of NAC in eradicating LN metastases

59 in a region-by-region manner for the first time.

60 
MATERIALS AND METHODS

\section{Assessment of MDCT images}

Patients

With the written consent of each patient, and under the approval of the ethics committee, we

initiated the MDCT study in January 2007: 106 patients underwent MDCT examination

within two weeks before surgery; 78 patients underwent primary surgery; and the remaining

28 patients received NAC followed by surgery. In Table 1, we show the number of patients

arranged according to their cancer stage.

Lymphadenectomy was performed during all of the surgeries, and we resected LNs

separately in a region-by-region manner and made pathological diagnoses for each sample.

$N A C$ regimen and indication

Because squamous cell carcinoma (SCC) was more sensitive than adenocarcinoma to

chemotherapy in our preliminary analysis, we administered NAC primarily in stage 
78 on days one and eight) and nedaplatin (NDP) $\left(80 \mathrm{mg} / \mathrm{m}^{2}\right.$ on day one) was administered.

79 Cycles were repeated every 21 days. In total, we conducted two cycles of NAC.

after the intraoperative pathological diagnosis of frozen sections or when the surgeon found it

necessary. LNs and their surrounding tissue were removed from the bifurcation of the

common iliac artery to either the inferior mesenteric artery (IMA) or the level of the renal vein.

supine position, and the whole abdomen and pelvis were scanned. Image data were acquired

with a $7 \mathrm{~mm}$ slice thickness, using a $64 \times 0.5 \mathrm{~mm}(64$-detector-row CT) or $16 \times 1.0 \mathrm{~mm}$ 

mm (64-detector-row CT) or $14.9 \mathrm{~mm}$ (16-detector-row CT) table feed per rotation. The acquired images were used to generate images with $1 \mathrm{~mm}$ slice thickness. All dual-phase contrast-enhanced CT images with a single breath-hold were obtained at 90 seconds following the intravenous injection of $100 \mathrm{ml}$ of $300 \mathrm{or} 350 \mathrm{mg} / \mathrm{ml}$ non-ionic contrast medium (Iomeron ${ }^{\circledR} 350$, Eisai, Tokyo, Japan; Iopamiron ${ }^{\circledR} 300$, Bayer HealthCare, Osaka, Japan; or Omnipaque ${ }^{\circledR} 300$, Daiichi-Sankyo, Tokyo, Japan) at a rate of $2.5 \mathrm{ml} / \mathrm{sec}$ using an automatic injector.

\section{radiation dose to the whole abdomen was approximately $10 \mathrm{mSv}$. The noise of the images}

with $1 \mathrm{~mm}$ thickness was greater than those with $7 \mathrm{~mm}$ thickness. However, there was no

difficulty in evaluating length of LNs, especially in MDCT images with contrast

enhancement.

\section{Evaluation of LNs by MDCT}

The evaluation of LNs using the MDCT images of $1 \mathrm{~mm}$-thick slices was performed independently by a radiologist (AK) who specializes in the field of gynecology and by a gynecologic oncologist (KY). The size of each LN, measured independently by AK and KY to the first decimal place, was determined by consensus of them. 
112

113

114 study, we aimed to analyze more closely. Because it is unfeasible to compare each LN

Region-by-region analysis

detected in MDCT images with LN detected in operation in node-by-node manner, we

employed a region-by-region analysis. In a previous study, the pelvic and para-aortic spaces

were divided into five regions prior to conducting a region-by-region analysis [9]. In the present study, we divided them into 13 regions (Supplementary Figure 1) to conduct a more detailed analysis. We determined the size of the largest $\mathrm{LN}$ in each region, termed the regional $\underline{\mathrm{LN}}(\underline{\operatorname{Reg}-\mathrm{LN}})$, and compared it with the pathological diagnosis of LN metastasis in each region. We should note that even if LN metastasis existed in a region, the Reg-LN might not be the involved LN. Receiver operating characteristic (ROC) curve were drawn to determine the optimal cut-off size. For the development of the new criterion, we analyzed only the primary operation cases and excluded the NAC cases.

Estimate of the efficacy of NAC in eradicating LN metastases using MDCT images

In patients who received NAC, we performed the MDCT examination before and 
$130 \quad$ Statistical methods

131 We used t-tests to compare the parametric values between two groups and one-way

132 ANOVA for three groups. Paired t-tests were used for the paired analysis. To analyze the

133 distribution in a $2 \times 2$ table, Fisher's exact test was employed. To analyze the distribution in a

134 larger table, the chi-square test was used. We considered a value of $p<0.05$ to be a significant

135 difference.

136 
RESULTS

Detection of LNs in $1 \mathrm{~mm}$ slice images from MDCT.

Only seven of the 800 visible LNs in the MDCT images were over $10 \mathrm{~mm}$ in diameter. regions than in the non-metastatic regions $(\mathrm{p}=0.006)$ (Figure $1 \mathrm{~b})$. In the following analyses, the largest $\mathrm{LN}$ in each region was defined as the Reg-LN.

Development of a new criterion to diagnose LN metastasis. 
analysis using the ROC curve is difficult when the number of metastasis-positive cases is less than five. Only the external iliac and obturator regions were positive for metastasis in five or more patients. Thus, we analyzed the Reg-LNs of the obturator region, external iliac region, and the other regions separately. In all three groups, the size of the Reg-LN was significantly larger in the LN regions containing metastases than in the non-metastatic regions; the mean was 7.9 in the metastatic case vs. $4.0 \mathrm{~mm}$ in the non-metastatic case in the external iliac $(\mathrm{p}<0.0001), 6.6$ vs. $3.9 \mathrm{~mm}$ in the obturator $(\mathrm{p}<0.0001)$, and 5.1 vs. $3.8 \mathrm{~mm}$ in the other regions $(\mathrm{p}=0.003)$ (Figure 2a). The variation in the size of the Reg-LNs in these three groups was not significant in either metastatic or non-metastatic regions (Figure 2a). The optimal cut-off sizes determined by the ROC curves in the external iliac, obturator, and other regions were $4.9,5.3$, and $5.1 \mathrm{~mm}$, respectively (Figure $2 \mathrm{~b}$ ). Based on these results, $5 \mathrm{~mm}$ was selected as the cut-off size for all types of LN regions. significantly larger in the LN regions containing metastases than in the regions without metastases: mean $6.4 \mathrm{~mm}$ vs. $3.9 \mathrm{~mm}(\mathrm{p}<0.0001)$ (Figure 3a). Based on the ROC curve, the optimal cut-off size to diagnose a LN metastasis was $4.7 \mathrm{~mm}$ (AUC $=0.80$, Figure $3 \mathrm{~b}$ ). In the 
clinical use. When the $5 \mathrm{~mm}$ cut-off was used, the sensitivity, specificity, positive predictive

value (PPV), and negative predictive value were 70, 79, 27, and 96\%, respectively.

Next, we divided all cases into SCC and non-SCC to examine the influence of

histological differences on the detection of LN metastasis. In both SCC and non-SCC patients,

the size of the Reg-LN was significantly larger in the LN regions with metastases than in the

$\mathrm{mm}(\mathrm{p}<0.0001)$ (Figure 3a). The size of the Reg-LN in non-metastatic LN regions was larger

in SCC compared with non-SCC, but the difference was small (Figure 3a). The optimal

cut-off determined by the ROC curve was $5.4 \mathrm{~mm}$ in SCC and $4.7 \mathrm{~mm}$ in non-SCC cases

(Figure 3c). Thus, $5 \mathrm{~mm}$, the closest whole number, was again selected for use in both

histological subtypes.

We also evaluated the conventional $10 \mathrm{~mm}$ cut-off. In the primary surgery cases, only

four of all the visible LNs in the MDCT images were over $10 \mathrm{~mm}$ in diameter (Figure 2a).

We found that the conventional cut-off of $10 \mathrm{~mm}$ was far from the optimal cut-off based on 
shown). We therefore concluded that the conventional $10 \mathrm{~mm}$ cut-off is statistically inappropriate in MDCT images.

\section{Assessment of NAC efficacy on metastatic LNs in cervical cancer.}

surgery using the MDCT images taken just before surgery. As in the primary surgery cases,

primary surgery groups (Figure 4a). Similar to the primary surgery cases (Figure 4b), the

optimal cut-off determined by the ROC curve was $4.7 \mathrm{~mm}$ in NAC cases $(\mathrm{AUC}=0.83$,

Figure 4c).

Next, we examined the size change of LNs after NAC. For this analysis,

node-by-node comparison was performed. First, we compared the size of all visible 328 LNs

individually between before and after NAC. NAC significantly decreased the size of LNs

from 5.1 to $3.5 \mathrm{~mm}(\mathrm{p}<0.0001)$ (Figure $5 \mathrm{a})$ : this is the reduction in the average size of all 
205 (p<0.0001) (Figure 5b). Using the cut-off of $5 \mathrm{~mm}, 80$ out of 158 Reg-LNs $(51 \%)$ were $5 \mathrm{~mm}$

206 or greater in diameter before NAC. After NAC, the number of Reg-LNs that were $5 \mathrm{~mm}$ or

207 greater was significantly decreased to $41(\mathrm{p}<0.0001,26 \%)$ (Figure $5 c$ ). Of these 41 regions,

$20813(32 \%)$ had LN metastases based on their pathology. Among 78 regions that were less than

$2095 \mathrm{~mm}$ in diameter before NAC, a LN metastasis was identified in only 1 region (1\%) after

$210 \quad$ NAC.

211

In addition to the analysis of MDCT images, we reviewed the medical records of

212 cervical cancer cases that were treated in our hospital between 1999 and 2011. In accordance

213 with the MDCT analysis, we found that NAC decreased the frequency of LN metastasis

214 (Supplementary text, Supplementary Figure 2b). 
DISCUSSION

The main objective of this study was to generate a new and more reliable criterion to

diagnose LN metastasis pre-operatively. We also evaluated the efficacy of NAC in

eradicating LN metastasis using the newly developed criterion because NAC has the potential

to eradicate $\mathrm{LN}$ and distant metastases $[2,15,16]$.

for the short-axis diameter in the diagnosis of swollen LNs as potential metastases. However,

only seven of 800 visible LNs in the MDCT images were over $10 \mathrm{~mm}$ in diameter in this

study (data not shown). Therefore, the conventional cut-off of $10 \mathrm{~mm}$ is not effective in

clinical practice. The conventional cut-off is used because of the limitations of conventional

CT, which cannot reproducibly measure an object smaller than $10 \mathrm{~mm}$ in diameter in $5-7 \mathrm{~mm}$ slices [7]. acquisition [18]. In addition, MDCT provides more information than CT without increasing 
oesophageal, breast and pancreatic cancer [10, 11, 20-22], to the best of our knowledge, this

234 is the first report on the use of MDCT in a gynecological cancer. of the LN metastasis is essential. Accordingly, we determined that the cut-off should be ascertained using region-by-region analysis. practice. In addition, a cut-off of $5 \mathrm{~mm}$ using MDCT was reproducible regardless of the location or pathological subtype of the tumor (Figures 3b, 3c, 4c). The ROC analyses clearly showed that a cut-off of $10 \mathrm{~mm}$ is not appropriate. metastasis in NAC cases was significantly lower than in primary surgery cases

248 (Supplementary Figure 2b). This result is consistent with previous reports that the frequency 
approximately half of the Reg-LNs that were $5 \mathrm{~mm}$ or larger shrank to less than $5 \mathrm{~mm}$ after

251 NAC (Figure 5c). Although shrinkage of LNs does not necessary mean anti-tumor effect

against involved LNs, this study shows a substantial number of involved LNs might have

NAC; however, further studies are required to confirm whether NAC is effective against the

LN metastasis of cervical cancer. involved region may not always be the involved LN. To compare the preoperative image with the pathological diagnosis in node-by-node manner, data accumulated from image-guided surgery, currently used for gastric or liver cancer [23, 24] are necessary. Although the detection rate of at least one $\mathrm{LN}$ in a region was increased when involved LNs exist, the detection rate was still $75 \%$, not $100 \%$ (Figure 1b). Among operable cervical caner patients, PPV of $5 \mathrm{~mm}$ cut-off was only $27 \%$ although PPV is thought to be higher in advanced cases where operation is impossible and radiotherapy is indicated. 
267 would be validated in a prospective study with a large number of cases and node-by-node

268 surgery with imaging analysis.

270 diagnosis of metastatic LNs within an LN region. This novel diagnostic criterion would

271 improve the individualized treatment of cervical cancer patients. In the future, clinical trials

272 should be performed to investigate whether this strategy could improve the prognosis of

273 cervical cancer patients.

275 Conflict of Interest Statement: All authors declare that they have no conflicts of interest.

276 Acknowledgments

277 We gratefully thank Dr. Takeshi Kubo for evaluating radiation dose of MDCT. 
280 [1] Rose PG. Chemoradiotherapy: the new standard care for invasive cervical cancer. Drugs.

2000;60(6):1239-44.

[2] Matsumura M, Takeshima N, Ota T, Omatsu K, Sakamoto K, Kawamata Y, et al.

283 Neoadjuvant chemotherapy followed by radical hysterectomy plus postoperative

284 chemotherapy but no radiotherapy for Stage IB2-IIB cervical cancer--irinotecan and platinum

285 chemotherapy. Gynecol Oncol. 2010;119(2):212-6.

286 [3] Tanaka Y, Sawada S, Murata T. Relationship between lymph node metastases and

287 prognosis in patients irradiated postoperatively for carcinoma of the uterine cervix. Acta

288 Radiol Oncol. 1984;23(6):455-9.

289 [4] Takeda N, Sakuragi N, Takeda M, Okamoto K, Kuwabara M, Negishi H, et al.

290 Multivariate analysis of histopathologic prognostic factors for invasive cervical cancer

treated with radical hysterectomy and systematic retroperitoneal lymphadenectomy. Acta

292 Obstet Gynecol Scand. 2002;81(12):1144-51.

293 [5] Aoki Y, Sasaki M, Watanabe M, Sato T, Tsuneki I, Aida H, et al. High-risk group in

294 node-positive patients with stage IB, IIA, and IIB cervical carcinoma after radical

295 hysterectomy and postoperative pelvic irradiation. Gynecol Oncol. 2000;77(2):305-9. 
296 [6] Hricak H, Yu KK. Radiology in invasive cervical cancer. AJR Am J Roentgenol.

$297 \quad 1996 ; 167(5): 1101-8$.

298 [7] Scheidler J, Hricak H, Yu KK, Subak L, Segal MR. Radiological evaluation of lymph

299 node metastases in patients with cervical cancer. A meta-analysis. Jama.

$300 \quad$ 1997;278(13):1096-101.

301 [8] Schwartz LH, Bogaerts J, Ford R, Shankar L, Therasse P, Gwyther S, et al. Evaluation of

302 lymph nodes with RECIST 1.1. Eur J Cancer. 2009;45(2):261-7.

303 [9] Choi HJ, Roh JW, Seo SS, Lee S, Kim JY, Kim SK, et al. Comparison of the accuracy of

304 magnetic resonance imaging and positron emission tomography/computed tomography in the

305 presurgical detection of lymph node metastases in patients with uterine cervical carcinoma: a

306 prospective study. Cancer. 2006;106(4):914-22.

307 [10] Yan C, Zhu ZG, Yan M, Zhang H, Pan ZL, Chen J, et al. Value of multidetector-row

computed tomography in the preoperative $\mathrm{T}$ and $\mathrm{N}$ staging of gastric carcinoma: a large-scale

309 Chinese study. J Surg Oncol. 2009;100(3):205-14.

310 [11] Kim AY, Kim HJ, Ha HK. Gastric cancer by multidetector row CT: preoperative staging.

311 Abdom Imaging. 2005;30(4):465-72. 
[12] Parkin DM, Bray F, Ferlay J, Pisani P. Estimating the world cancer burden: Globocan

2000. Int J Cancer. 2001;94(2):153-6.

[13] Piver MS, Rutledge F, Smith JP. Five classes of extended hysterectomy for women with

cervical cancer. Obstet Gynecol. 1974;44(2):265-72.

CT radiation dose reduction: how to implement change without sacrificing diagnostic quality.

[15] Rydzewska L, Tierney J, Vale CL, Symonds PR. Neoadjuvant chemotherapy plus surgery versus surgery for cervical cancer. Cochrane Database Syst Rev. 2010(1):Cd007406.

[16] Cai HB, Chen HZ, Yin HH. Randomized study of preoperative chemotherapy versus primary surgery for stage IB cervical cancer. J Obstet Gynaecol Res. 2006;32(3):315-23. tomography and magnetic resonance imaging in staging of uterine cervical carcinoma: a systematic review. Gynecol Oncol. 2003;91(1):59-66. 
[19] Lawler LP, Fishman EK. Multi-detector row CT of thoracic disease with emphasis on

3D volume rendering and CT angiography. Radiographics. 2001;21(5):1257-73.

[20] Lin H, Chen TW, Li ZL, Zhang XM, Chen XL, Wang LY, et al. Tumor size of resectable the assessment of axillary lymph nodes before surgery for breast cancer using multidetector-row computed tomography. Surg Today. 2010;40(11):1023-6. Preoperative diagnosis of lymph node metastasis in biliary and pancreatic carcinomas: evaluation of the combination of multi-detector CT and serum CA 19-9 level. Dig Dis Sci. 2010;55(12):3617-26.

[23] Kim YM, Baek SE, Lim JS, Hyung WJ. Clinical application of image-enhanced minimally invasive robotic surgery for gastric cancer: a prospective observational study. $\mathbf{J}$

Gastrointest Surg. 2013;17(2):304-12. 
$346 \quad$ 2010;65(4):463-78.

347 
349 Figure 1. Detection of LNs in MDCT images. a) The number of detected LNs by MDCT is

350 indicated by a black box. Pathologically detected LNs are indicated by the sum of the detected and undetected LNs. The distribution was statistically significant $(\mathrm{p}<0.0001$, chi-square test). PAN: para-aortic LN region; IMA: inferior mesenteric artery; Bif: aortic bifurcation; L: left; R: right. b) The detection rate of Reg-LNs was significantly higher in the involved regions $(\mathrm{pN} 1)$ than in the uninvolved regions $(\mathrm{pN} 0)(\mathrm{p}=0.006)$.

Figure 2. Region-by-region analysis of LNs. a) Each dot indicates the size of the Reg-LN. Red dots indicate size of involved Reg-LNs and tiny black dots indicate size of uninvolved Reg-LNs. Black bar indicates the mean size. Ext: external iliac region; Obt: obturator region; Others: all other regions. One-way ANOVA was used for the statistical analysis. b) ROC curves based on the size of the Reg-LN and the presence of the LN metastasis. The gray arrowhead indicates the optimal cut-off based on the ROC curves. Note that the $10 \mathrm{~mm}$ cut-off indicated by the black arrowhead is away from the optimal cut-off based on the ROC analysis.

Figure 3. Region-by-region analysis of all Reg-LNs among different histological subtypes. a)

364 Each dot indicates the size of the Reg-LN. Red dots indicate size of involved Reg-LNs and 
tiny black dots indicate size of uninvolved Reg-LNs. Black bar indicates the mean size. To

detect the association of all Reg-LNs with the different histological subtypes present in the

three groups, one-way ANOVA was performed. b) ROC curves are based on the size of the

Reg-LN and the presence of the LN metastases. c) ROC curve analyses were conducted by

dividing the cases into different histological subtypes. The gray arrowhead indicates the

arrowhead is away from the optimal cut-off based on the ROC analysis.

Figure 4. Region-by-region analysis for primary surgery cases and NAC cases, and the effect of NAC based on the analysis of MDCT images. a) Each dot indicates the size of the Reg-LN.

374 Primary: primary surgery; pN0: no LN metastasis; pN1: LN metastasis; NS: not statistically significant. b) ROC curves are based on the size of the Reg-LN and the presence of the LN metastasis in all primary surgery cases. The gray arrowhead indicates the optimal cut-off based on the ROC curves. c) ROC curve representing only the NAC cases. The cut-off in the

NAC cases was $4.7 \mathrm{~mm}$.

Figure 5. Effect of NAC according to MDCT images. a) Changes in all LN sizes that were 
decreasing the proportion of regions with a LN larger than $5 \mathrm{~mm}$ (black bar). Red boxes

indicate regions with $\mathrm{LN}$ metastasis. NAC decreased the number of regions with LNs larger after NAC, we found $6(5 \%)$ involved regions.

Supplementary Figure 1. We divided para-aortic and pelvic lymph nodes into 13 regions to compare pre-operative images with the pathological diagnosis. a) para-aortic lymph nodes, from IMA to renal vein; b) para-aortic lymph nodes, from bifurcation to IMA; c) right common iliac lymph nodes; d) left common iliac lymph nodes; e) presacral lymph nodes; f) right external iliac lymph nodes; g) right internal iliac lymph nodes; h) left internal iliac lymph nodes; i) left external iliac lymph nodes; j) right superficial inguinal lymph nodes; k) lymph nodes in the right obturator fossa (obturator, carinal, uterine artery, and deep inguinal lymph nodes); 1) lymph nodes in the left obturator fossa; m) left superficial inguinal lymph nodes.

Supplementary Figure 2. a) Changes in the treatment modality for SCC cases in our 
399 stage IB2/IIA SCC patients. c) Among IB2/IIA cases, NAC-treated cases showed a tendency

400 toward better survival compared with the primary operation cases, although this result was

401 not statistically significant.

402 
403 TABLE

404

405 Table 1. Number of patients multi-detector CT image was analyzed.

406

\begin{tabular}{cccc}
\hline Stage & SCC & Non-SCC & Total \\
\hline IA & 4 & 3 & 7 \\
IB1 & 32 & 20 & 52 \\
IB2 & $10(7)$ & $4(1)$ & $14(8)$ \\
IIA & 4 & 1 & 5 \\
IIB & $23(19)$ & $5(1)$ & $28(20)$ \\
\hline Total & $73(26)$ & $33(2)$ & $106(28)$ \\
\hline
\end{tabular}

407

( ); NAC cases.

408 


\section{Figure 1}

\section{a}

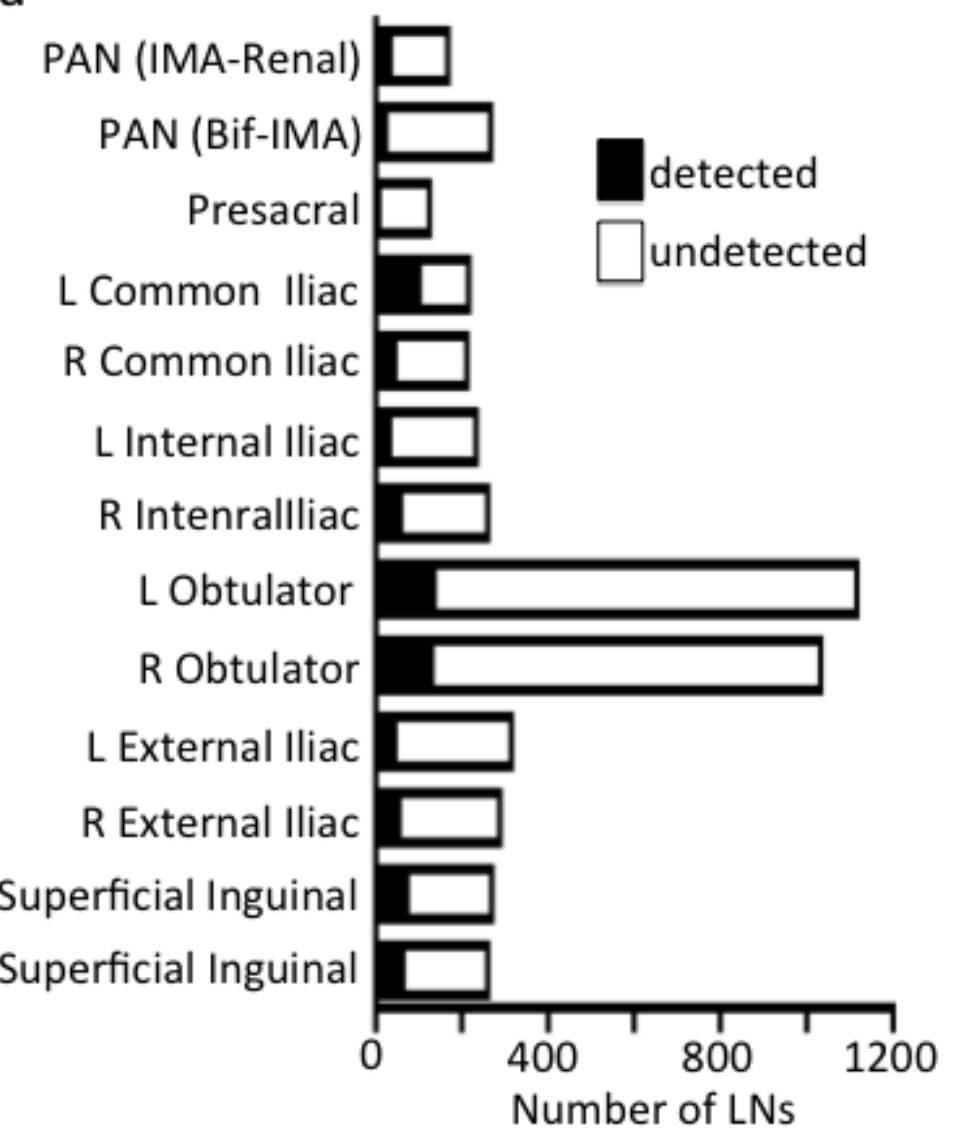

b


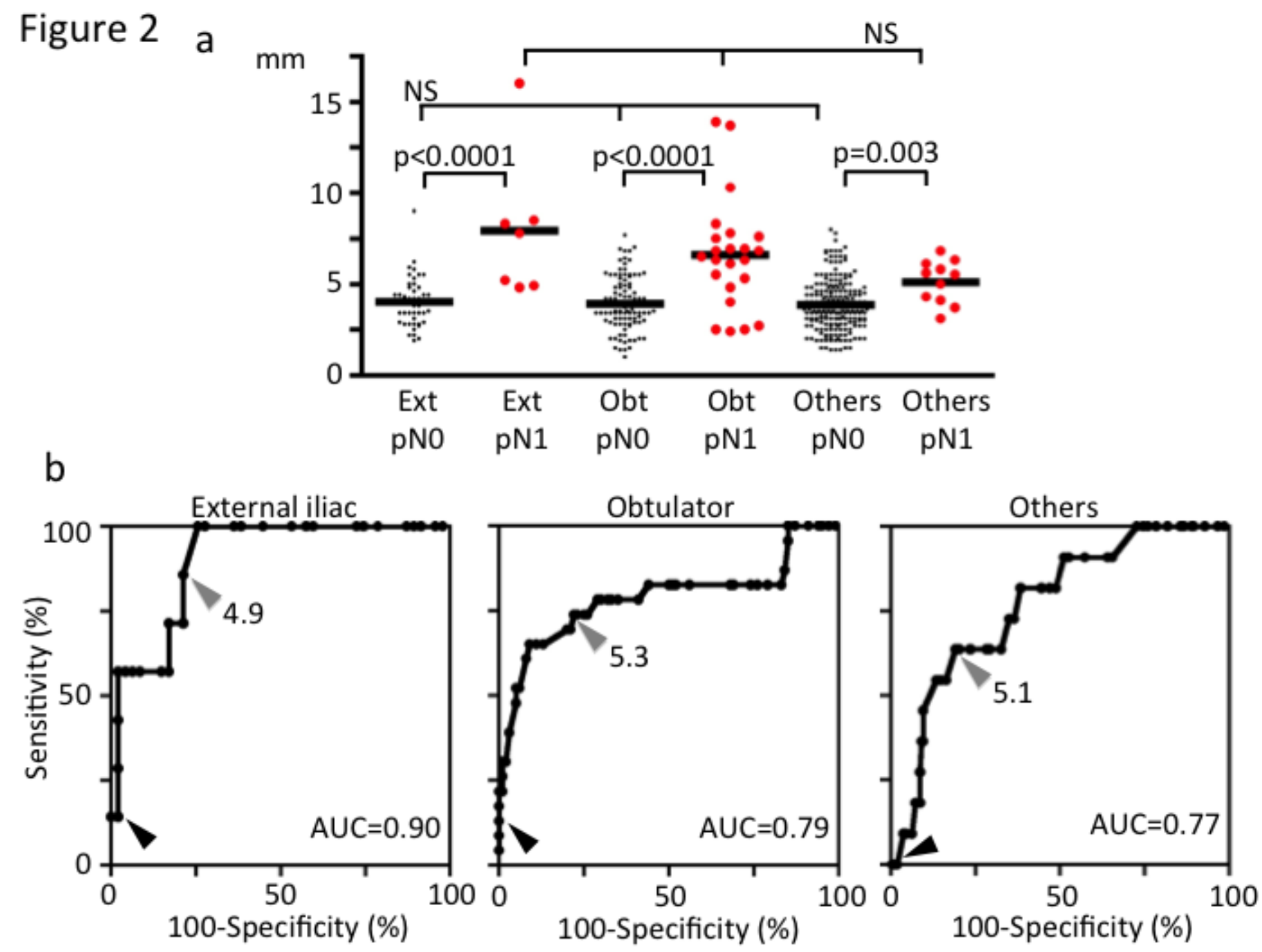


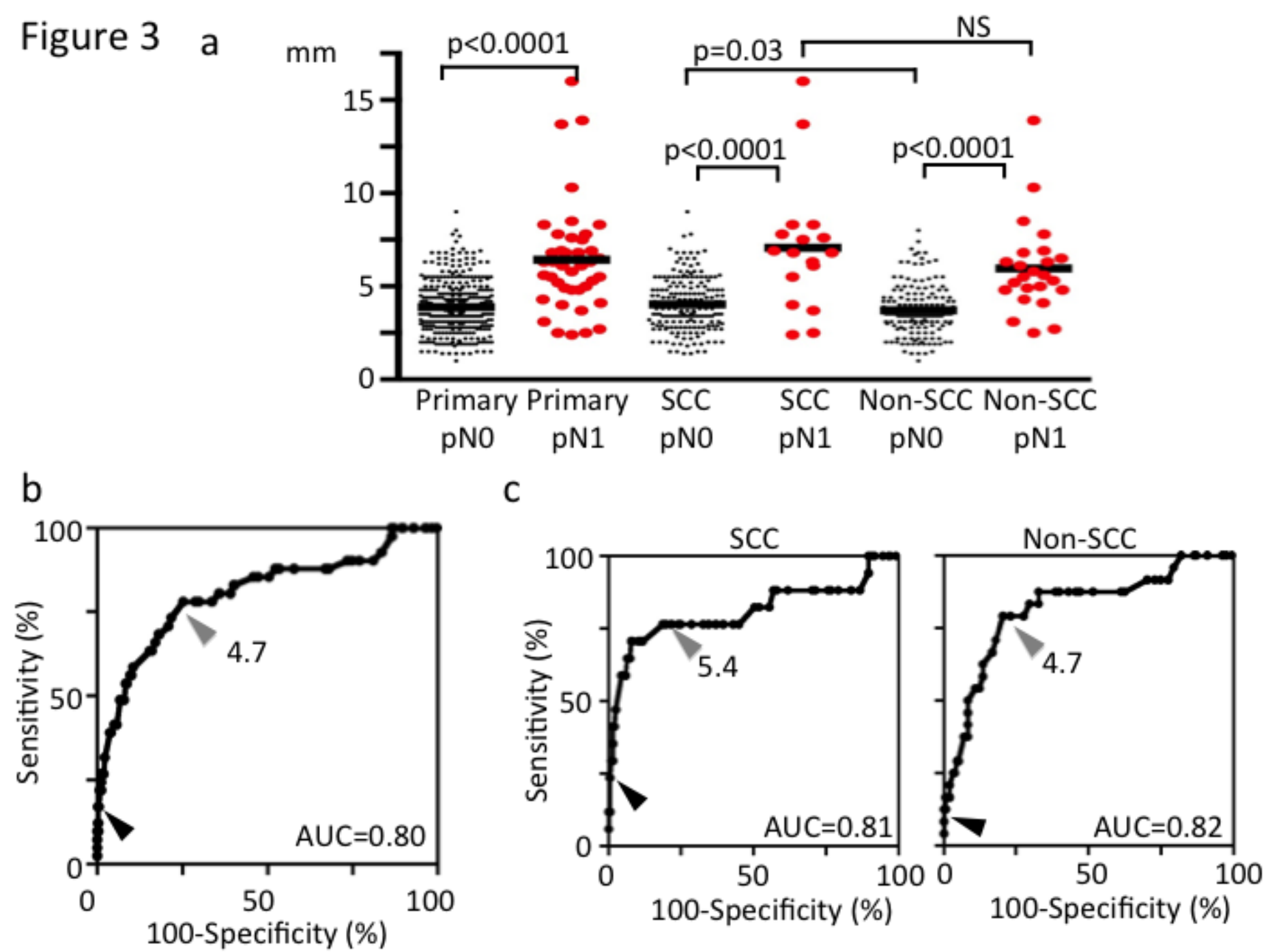


Figure 4

a

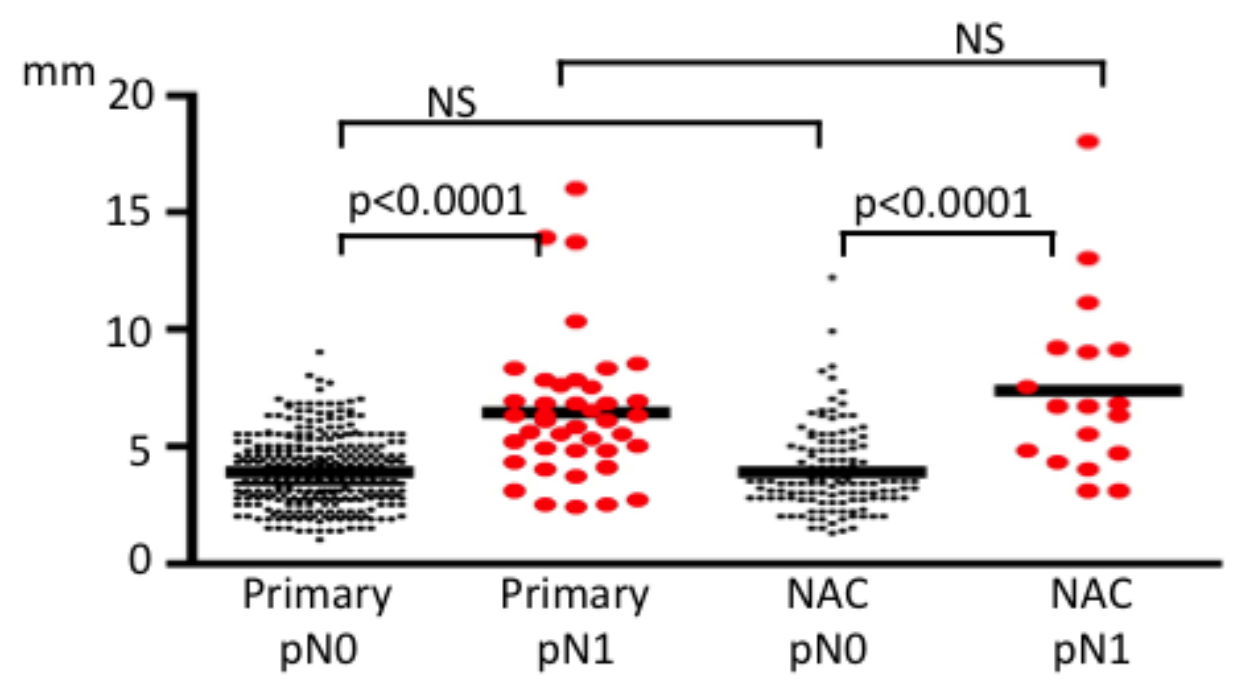

b
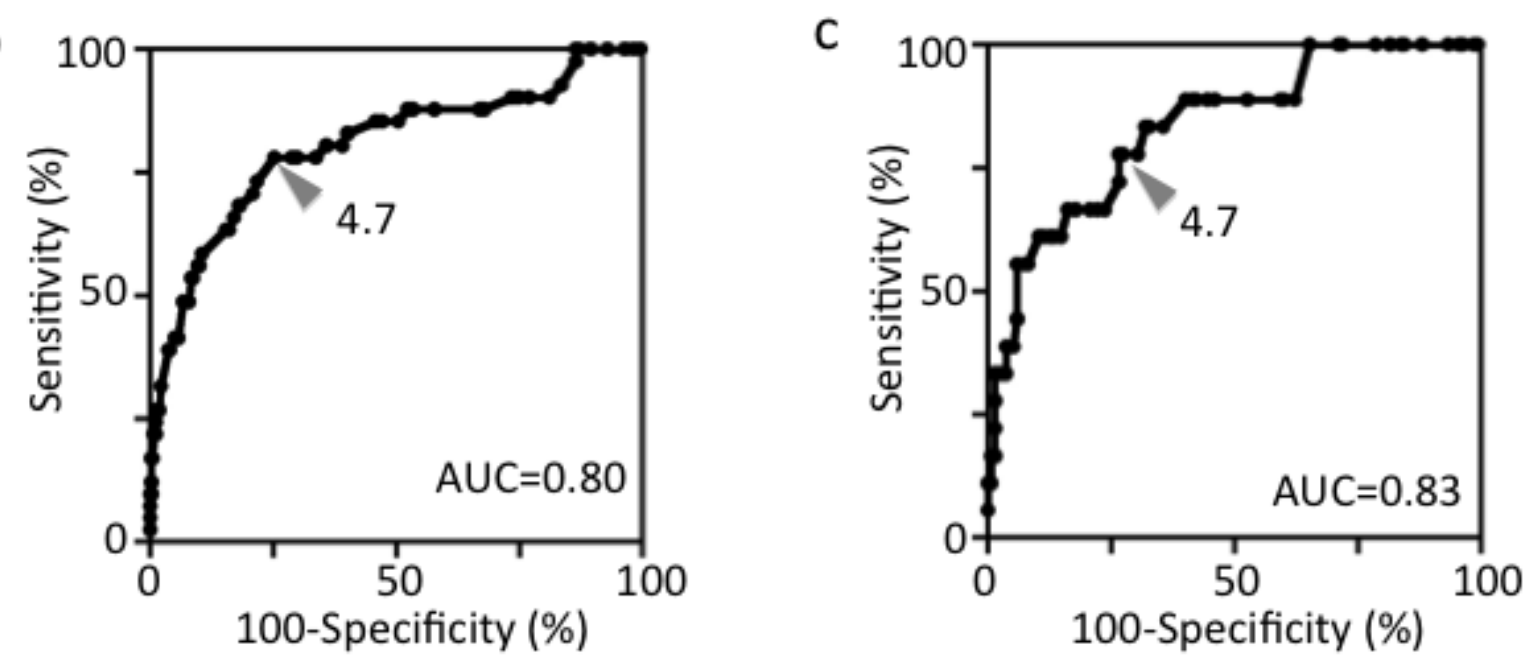
Figure 5

a

$\mathrm{mm}$

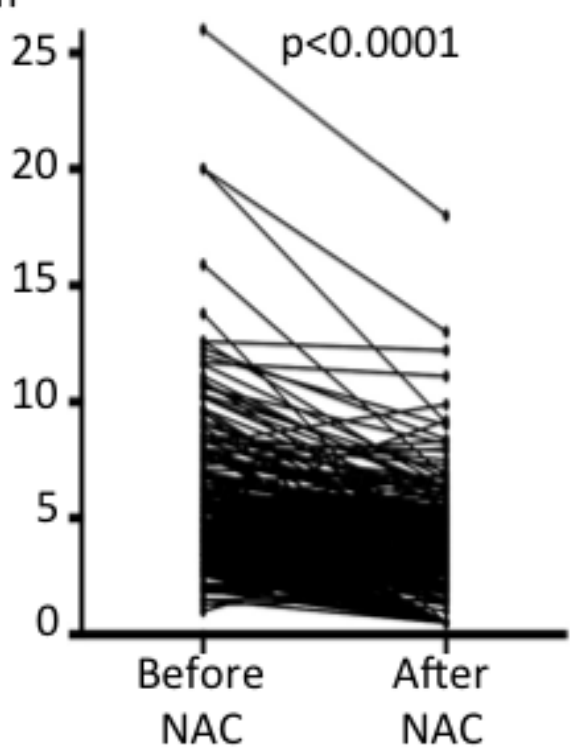

b $\mathrm{mm}$

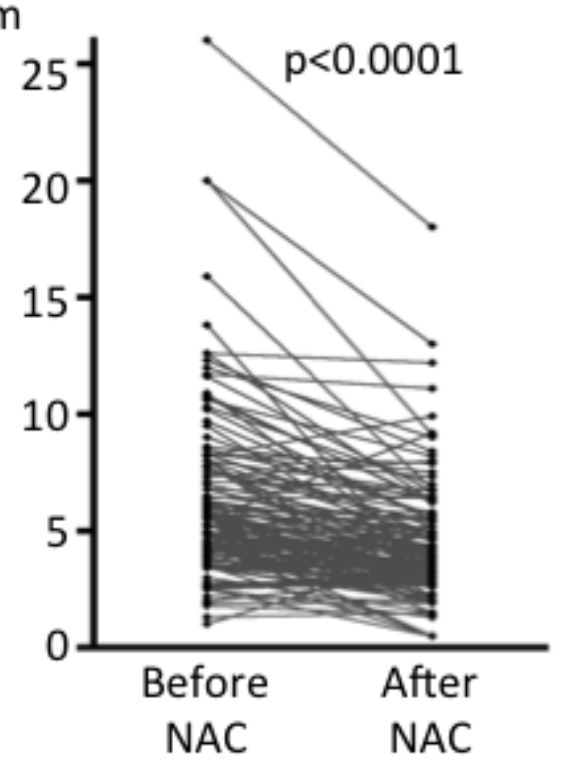

C

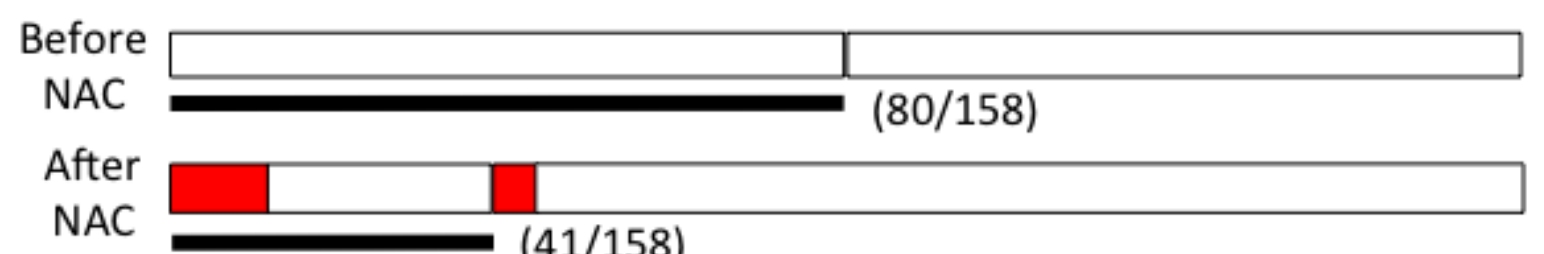

' $\mathrm{pN}(+) \longrightarrow 5 \mathrm{~mm}$ 


\section{Supplementary Text}

\section{Historical analysis of the effect of NAC in eradicating LN metastases}

\section{MATERIALS AND METHODS}

\section{Patients}

With the written consent of each patient and the approval of our ethics committee, we retrospectively reviewed the medical records of 296 cervical cancer cases (stage IA, IB, and IIB) who underwent systematic lymphadenectomy at our department between 1999 and 2011. The median number of resected pelvic lymph nodes was $44 \pm 38$ (mean \pm 2 SD) per patient. A total of 199 were diagnosed with SCC and 97 with non-SCC (adenocarcinoma, adenosquamous carcinoma, etc.) (Supplementary Table 1).

NAC regimen and indication

NAC regimens are shown in Supplementary table 2. Between 1999 and 2006, the most frequently used regimen was transarterial infusion of cisplatin (CDDP), (100 $\left.\mathrm{mg} / \mathrm{m}^{2}\right)$, pirarubicin (THP), $\left(25 \mathrm{mg} / \mathrm{m}^{2}\right)$, and mitomycin-C (MMC), (20 mg/patient) 
every 28 days. Between 2007 and 2011, intravenous infusion of irinotecan (CPT-11), $\left(60 \mathrm{mg} / \mathrm{m}^{2}\right.$ on days 1 and 8$)$ and nedaplatin (NDP), $\left(80 \mathrm{mg} / \mathrm{m}^{2}\right.$ on day 1$)$ every 21 days was most frequently used. In principle, we conducted 2 cycles of NAC.

Because squamous cell carcinoma (SCC) was more sensitive than adenocarcinoma to chemotherapy in our preliminary analysis, we administered NAC primarily in SCC cases. As a result, most NAC cases (57/63) were SCC. From 1999 to 2011, for most stage IIB SCC patients, we administered NAC before operation. For stage IB2/IIA SCC patients, until 2006, we administered NAC for only those who had very bulky tumours, and most cases underwent primary operation. In 2007 , we changed our policy and have therefore administered NAC for most IB2/IIA SCC cases. As a result, the proportion of stage IB2/IIA patients who received NAC increased significantly from $4 / 29$ (14\% through 2006) to $10 / 17$ (59\%, 2007 onward) (supplementary Figure 2a). Therefore, the primary operation and NAC were comparable among IB2/IIA cases. 
To analyse the distribution in a $2 \times 2$ table, Fisher's exact test was employed. To analyse the distribution in a larger table, the chi-square test was used. We considered $\mathrm{p}<0.05$ a significant difference.

\section{RESULTS}

Of 296 patients receiving lymphadenectomy from 1999 to 2011, 63 received NAC. Response rate (complete response or partial response) of NAC was 91\% (52/57). Because we changed our treatment policy for stage IB2/IIA SCC cases in 2007, we compared the rate of pathologically positive LN metastases in stage IB2/IIA SCC cases between the period of 1999-2006 and the period of 2007-2011. The rate of LN metastasis decreased from $41 \%$ (12/29, through 2006) to $24 \%$ (4/17, 2007 onward), although this difference was not statistically significant. Dividing all IB2/IIA SCC cases into NAC and primary surgery groups, the LN metastasis rate was significantly lower in NAC than in the primary surgery group $(1 / 14,7 \%$ vs $15 / 32,47 \%)(\mathrm{p}=0.02)$ (Supplementary Figure 2b). The overall survival rate of IB2/IIA SCC patients who received NAC was better than primary operation cases (Supplementary Figure 2c). 


\section{SUPPLEMENTARY TABLE}

Supplementary Table 1: Number of patients receiving lymphadenectomy from 1999 to 2011.

\begin{tabular}{cccc}
\hline Stage & SCC & Non-SCC & Total \\
\hline IA & 14 & 7 & 21 \\
IB1 & 87 & $56(1)$ & $141(1)$ \\
IB2 & $28(11)$ & $18(1)$ & $46(12)$ \\
IIA & $16(2)$ & $4(1)$ & $20(3)$ \\
IIB & $54(43)$ & $12(4)$ & $66(47)$ \\
\hline Total & $199(56)$ & $97(7)$ & $296(63)$ \\
\hline
\end{tabular}

( ); NAC cases.

Supplementary Table 2: NAC regimen. Numbers of patients are shown in the table.

\begin{tabular}{c|ccc|c}
\hline & stage IB2 & stage IIA & stage IIB & Total \\
\hline CPT-11/NDP & 9 & 0 & 22 & 31 \\
CDDP/THP/MMC * & 2 & 2 & 15 & 19 \\
NDP/Peplomycin/Ifomide & 1 & 1 & 4 & 6 \\
CPT-11/CDDP & 1 & 0 & 1 & 2 \\
Taxol/Carboplatin & 0 & 0 & 4 & 4 \\
Docetaxel/Carboplatin & 0 & 0 & 1 & 1 \\
\hline
\end{tabular}

*Transarterial infusion. 


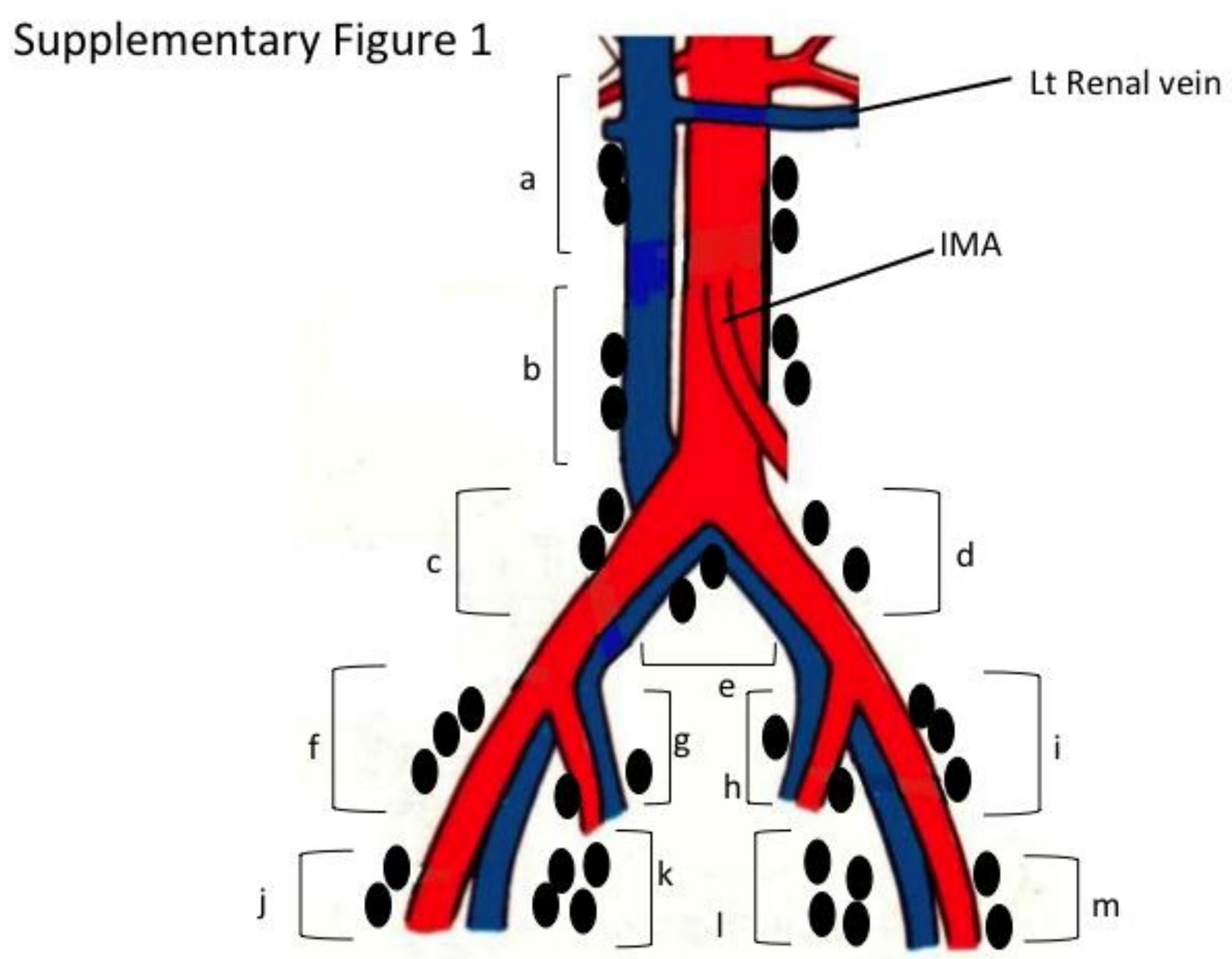




\section{Supplementary Figure 2}

a

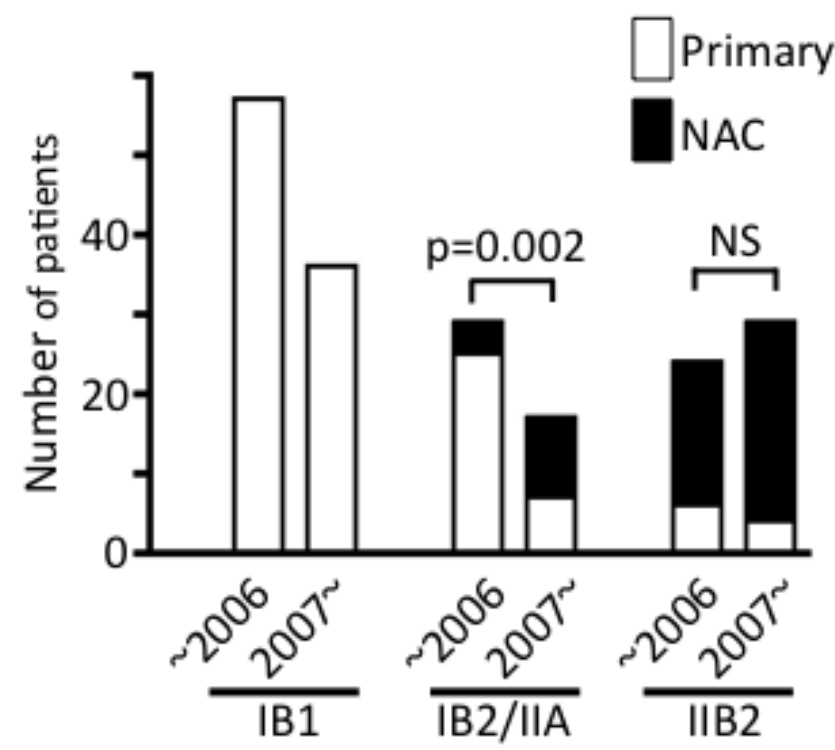

b
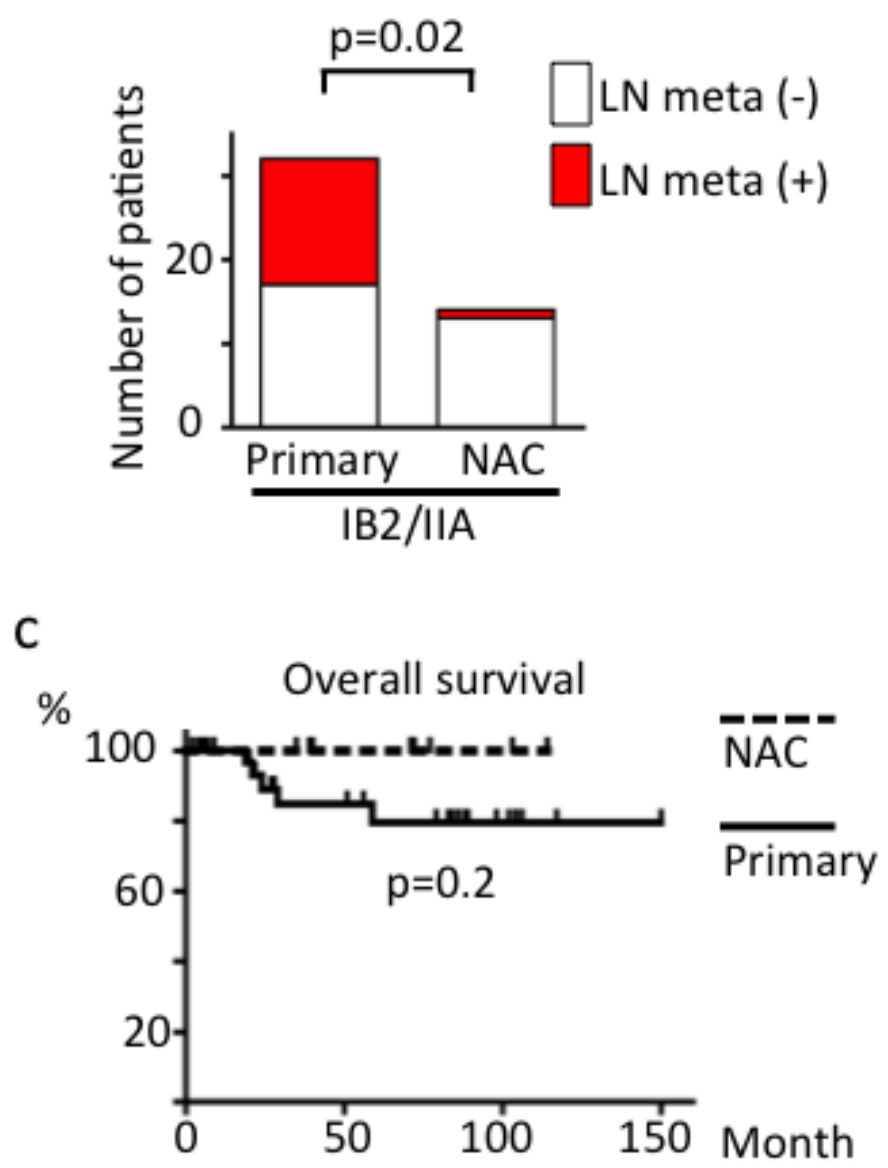